\title{
Implementation of "Virtual Studio Online" Desktop
}

\author{
Ruoyun Zhou, Chu Qiu, Quan Qi, Bo Wang, Yongquan Lu \\ High Performance Computing Center, Communication University of China, Beijing 100024, China \\ guring81@gmail.com, chqiu@cuc.edu.cn, yqlu@cuc.edu.cn
}

\begin{abstract}
This paper describes the implementation of "Virtual Studio Online" (VSO) desktop, a virtual desktop system integrating virtualization with high performance computing. Through the plugin mechanism, the hybrid virtualization framework of VSO desktop solves the problem of mixed virtualized environment management and automatic deployment. In the system, shared GPU and high performance computing cluster assures that users can obtain more powerful workstations than the local ones. While parallel storage system solves the lacking troubles in sharing hardware and software resources in VSO platform. In addition, web conference and onlineteam modules are also integrated to provide solutions for remote office and online creation teamwork. The VSO desktop system have found a wide variety of practical applications in our VSO platform, and good results have been achieved in actual practice.
\end{abstract}

Index Terms - virtual desktop; cloud computing; online creation.

\section{Introduction}

Cultural and creative industry is considered as one of the world's most promising industries in the 21 st century. Governments around the world have given the special attention to the development of this industry because of its great economic and social benefits. As an important approach and carrier for scale and intensive development of cultural and creative industry, cultural and creative industrial park has a strong momentum of construction. Yet, behind the rapid development of each park, some problems are also highlighted. For instance, too much parks make resources scattered; identical construction mode makes the park more estate like; high rent and low enterprises settled rate is difficult to attract the creative class, which makes the park culture weakness after the government spent heavily on the completion of the park, etc. As a result, creative industry chain cannot get effective integration and extension, and its value is not fully implemented.

Meanwhile, unprecedented changes have occurred in WEB since the information age, the effects that network brings to people's live is beyond their expectations. The emergence of cloud computing combines virtual technology with network, which achieves the concept of thin client, ensures that users can access to the high computing capability cloud at any time and any place using any terminal. Cloud computing not only make profound effects on software and operating system, but also had a huge impact to the cultural and creative industry at the same time. Combined with cloud computing and the cultural creative industry, the concept of "Virtual Studio Online" platform arise at the historic moment. VSO serves as an online creative industrial park for the cultural and creative industry, which breaks the physical and geographical limitations of the entity industrial park and greatly reducing the construction cost, provides an on-line creation virtual cloud platform for corporates and individuals working on cultural and creative industry [1].

VSO desktop, as a part of the VSO platform, provides users with a virtual environment that brings application services, resource sharing and collaborate teamwork together. Users are able to access the hardware and software resources that platform provides through the network in order to accomplish their work. In the past, due to the complexity of 3D animation scenes and special effect production, even if the designer has good inspiration, the production process still needs large hardware resources and long computation time. Moreover, a variety of software services support and teamwork is also required for further production. Speed and resource has become the main bottleneck for the development of cultural and creative workers. In the VSO virtual studio, users log on their studios through the VSO desktop and start using its customized services. Users can get their highperformance workstation with variety of configurations and computing resources, and create their own professional studio at any time. At the same time, by the manner of participating in other users studio, VSO desktop ensures that everyone can cooperate with others when using its platform. Also, with advanced parallel storage system, it gives users a secure studio environment that supports high availability and fault tolerance mechanism.

The realization of VSO desktop ensures the real-time interaction between users and resources, user and the platform, as well as users and users in VSO platform. Compared to traditional studio, virtual studio makes remote collaboration, home office possible. It not only accelerate the pace of cultural creation, shorten the creative cycles, but also save substantial investment for hardware and software equipment, thereby significantly reducing the costs of creative design, speed up the transformation process form cultural and creative ideas to economic benefits.

\section{Implementation of VSO Desktop}

Generally speaking, virtual desktop refers to Web Desktop (or Webtop [2]), which is running in the browser. However, VSO desktop is a concrete realization of the virtual studio, namely, present user customization of virtual resources with the form of virtual desktop. VSO desktop can unite web applications, web services, application server and the local client application into a desktop environment. It guarantees that users can get their customized high-performance workstation with a cross-platform thin client. 


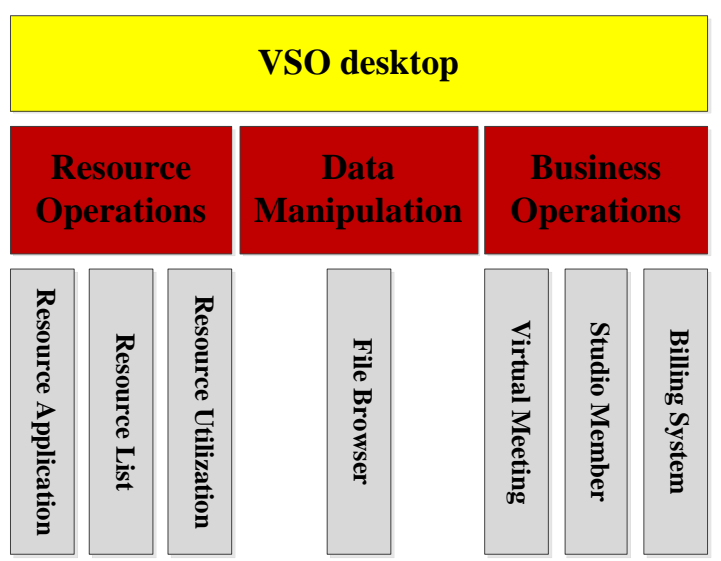

Fig. 1 VSO desktop client functional block diagram.

In Fig. 1, VSO desktop function module is divided into resource operations, data manipulation and business operations. Resource operation module is designed to meet the functional requirements of high-performance workstation automate distribution. Compared with traditional virtual desktop, which is lack of cloud virtual device resource management, VSO desktop build a new generation of infrastructure cloud management platform. This solution uses plug-in mechanism so that it merges the mature open source products and third-party commercial modules into a single management console. By combining browser-side file browsing operation function with cloud parallel storage system, data operation module provides users with a secure data center management capability. Business operations module implements participation function that allows users to join others' studio by logging on VSO desktop client. And through the web conference functions business operations module achieves real-time online communication and collaboration.

\section{A. Resource Operations}

\section{a) Resource Application}

VSO desktop provides users with a simple resource application process. For practitioners in the cultural and creative industry, different industries demand for diverse computing power resources. Resource application function in VSO desktop provides quantifiable resource information for each user. Users can choose computational resources according to their needs, and submit their customized cloud requests to the cloud through the application interface (Fig. 2).

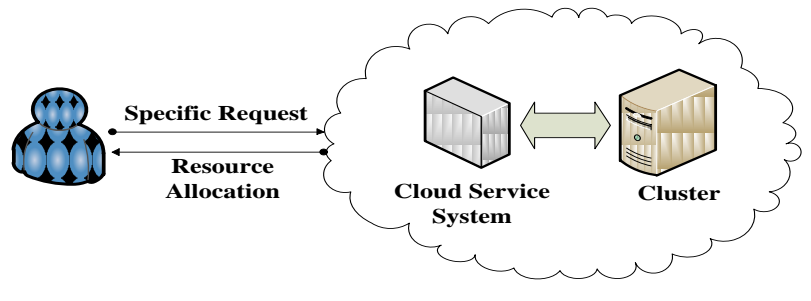

Fig. 2 Resources request-allocation process.
On the cloud, VSO desktop deploys a set of automation cloud service system for users. From automated application and configuration management for physical system or virtual machine deployment according to user requirements, to automatically unloaded configuration for freeing computing resource, cloud service system can provide a completely automated configuration cycle. Configuration and interfaces of cloud service system add in this solution with the form of cloud plug-in (Fig. 3), which provides fully automatic configuration and deployment cycle. Users from external data center can submit clouds requests through the second web portal on the server. Cloud request will fully automated configuration and deployment after manual or automatic approval.

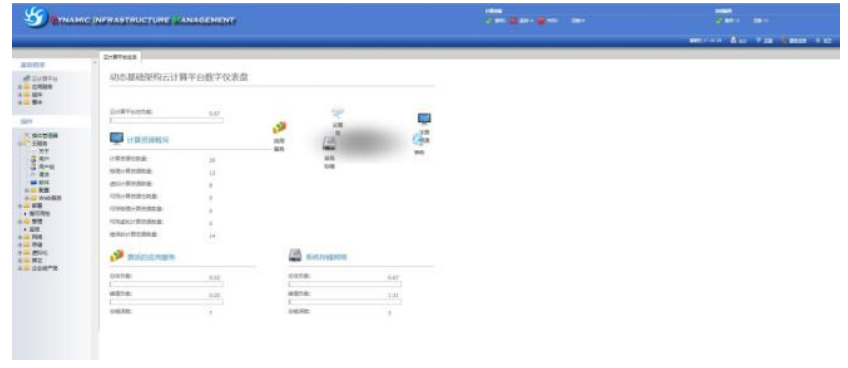

Fig. 3 Cloud service system dashboard.

Cloud service system provides system administrators with a governmental end that directly insert into system server. It provides a fine-grained overview, for current cloud activities and several configuration options, to adjust the system. Cloud service system, for example, can be set to auto or manual approve new cloud system request; will automatically create a new virtual machine if there is not enough available virtual machine; can enable or disable deployment clone function, etc. In addition, it also includes cloud system IP Manager to automatically configure the external network interface of configured device. By integrating the above features, this system is capable of skipping the complex detection process and achieving the goal of resources dynamic allocation.

\section{b) Resource List}

In the resource list, user can access history information of all resources, and be able to startup, shutdown, suspend, log as well as doing other operations to the virtual device in the list. With the transform from virtual equipment operation to the operating concept that matches the actual device allows user to understand easily. In VSO desktop, physical information and virtual information is integrated as a whole to provide services for users with the form of appliance service. Appliance service layer lies in the top of the VSO desktop architecture hierarchy (Fig. 4). For external it provide services for users, for internal it used to manage different system plugins. And management of physical infrastructure resource and virtual infrastructure resource (e.g., computing resources, storage resources, network resources, etc.) is implemented through plugins. VSO desktop architecture level can be logically divided into appliance service, system plugin and infrastructure. 


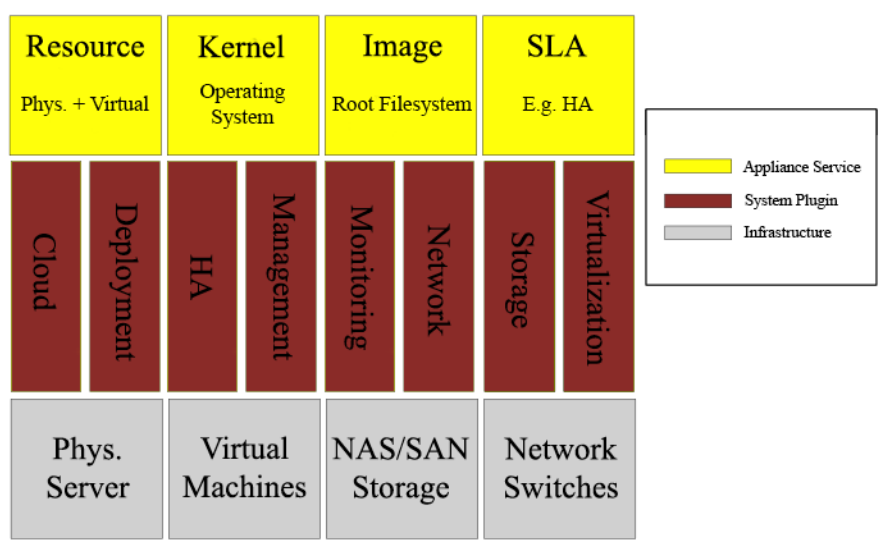

Fig. 4 Hierarchical graph of VSO desktop architecture.

Infrastructure layer provides the physical basis for VSO desktop, system plugin layer achieved pluggable management mode via the form of plugins, appliance service represents one (or more) of the actual services which should be provided by the datacenter. An appliance is the combination of a kernel, an (server-) image, a resource and service requirements plus service-level-agreements (SLA). With those requirements VSO desktop then fully automates the management of the specific services running on the appliance's server-image. Therefore, this hybrid virtualization framework solves the problem of mixed virtualized environment management, and complete IT infrastructure needs, such as automation, rapid deployment, real-time monitoring and high availability. It helps cloud managers unified management of data center operations, significantly reducing costs of data center management.

\section{c) Resource Utilization}

By periodically refresh technology, VSO desktop can obtain real-time status of cloud virtual device and push it to the user with the common desktop form (Fig. 5). Meanwhile, users can operate the running equipment and viewing device information through the browser.

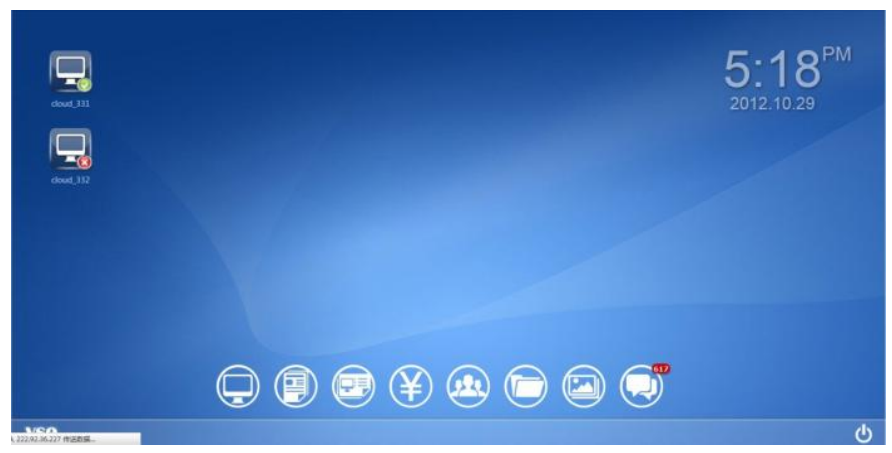

Fig. 5 VSO desktop interface.

Users can $\log$ on the active virtual resources in the form of remote desktop. Accordingly, desktop display protocol that affects virtual desktop user experience crucially. Current mainstream display protocol, including PCoIP, RDP, SPICE,
ICA, etc., is supported by different vendors. VSO desktop provides cross-device and cross-vendor desktop display protocol by integrating a web-based Remote Desktop Gateway. VSO desktop provides a Remote Desktop Protocol, which is similar to VNC and RDP protocol, for entering the desktop environment. As a tunnel or proxy, VSO desktop allows users to use a web browser to access multiple types of virtual desktop. And without installing any browser plugins and client programs, users only need a browser that supports HTML5 and AJAX to access virtual device in the VSO desktop on any device. That achieves the convenient login and cross-platform feature.

\section{B. Data Manipulation}

By combining browser-side file browsing operation function with cloud parallel storage system, data operation module provides users with a secure data center management capability. Parallel storage system introduces an object-based parallel storage technology, which uses common equipment to build powerful distributed storage system. In ensuring the normal file storage capabilities, swap space supply and other temporary storage function, system provides high-performance extended input and output with the design of object-based storage system architecture. Through RAID disk array technology in hardware, as well as hot-standby technology in software, system achieves high availability and fault tolerance mechanism of large-scale storage system. Thus it solves the conflict of parallel file access in distributed file system and ensures the security of storage system. Distributed caching system of which can effectively reduce the files reading and writing time, improve overall input/output performance and reduce latency.

\section{Business Operations}

\section{a) Virtual Meeting}

Web conferencing system provides an online communication platform for members from the same studio. It achieves real-time communication and collaboration, makes remote collaboration and home office possible. Web conferencing system provides users with the following features: record and playback, whiteboard, desktop sharing, integrated VoIP, presentation and web cam.

i. Record and Playback: System can record lectures for later playback by studio members.

ii. Whiteboard: Whiteboard controls let users annotate and call out key parts of their presentation for viewers.

iii. Desktop Sharing: go beyond slides, the presenter can broadcast their desktop for all studio members to see.

$i v$. Integrated VoIP: System voice conferencing supports voice over IP (VOIP) conferencing out-of-the-box. All studio members require are speakers and a microphone to participate.

v. Presentation: The presenter can upload any PDF presentation or Microsoft office document to present, zoom, pan, and keep studio members in sync. 
vi. Web Cam: Multiple users can share their webcam at the same time. There is no built-in limit on the number of simultaneously active webcams.

\section{b) Studio Member}

The implement of "join studio" logic in VSO desktop integrates numbers of individuals into a team rapidly, achieves a flexible flow of talent and greatly reduces the cost of a professional team.

Studio creators can search required talent in VSO talent pool and send the invitation to the talent. When accepting an invitation and joining the creator's studio, the talent have permissions to access creator's studio for resource application and eventually brings everyone's work together to form products through shared storage.

\section{c) Billing System}

Billing system is in communication with the cloud billing system, and be able to show consumed CCU and billing information (Fig. 6). Cloud service system equipped with imploded billing system, for calculating the amount of computing resources consumed by each user. Based on "cloud computing unit" (CCU) - virtual currency in the system, system administrators and users can plan and record their use of computing power and cost. Through the billing system, computing power is fully open to the final users with other methods on the market. And billing information pushes to users in order to achieve transparent management operations.

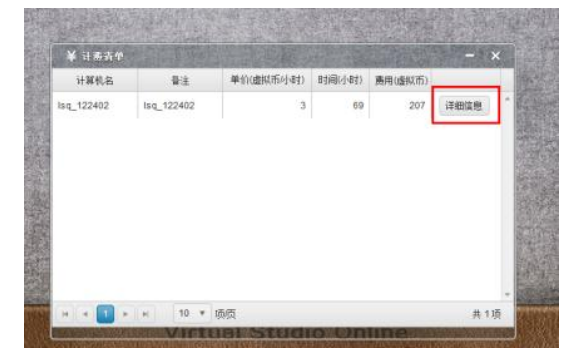

Fig. 6 Bill information function interface.

\section{Conclusion}

We have presented, VSO desktop, a system that provides users an integration service of resource access and resource operation. Combines resource operations, data manipulation and business operations, VSO desktop is able to provide cloud platform users with close to or even beyond the user experience that traditional local computer desktop can obtain. Moreover, VSO desktop realizes seamless access to users' virtual desktop services anytime and anywhere, gives users a convenient platform for transparent use of resources, and achieves a conflict-free and secure environments for resource sharing and collaborative.

\section{Acknowledgment}

The authors acknowledge the financial supports by the National Key Technology Support Program (2012BAH17B03) and the Program Project of CUC (XNG1138, YXJS2012319 and YXJS2012206).

\section{References}

[1] Yongquan Lu, Pengdong Gao, Chu Qiu, Jintao Wang, "A service cloud platform based on high-performance computing," China Science and Technology Achievements, vol. 12, no. 10, pp. 520-531, 2011

[2] Shaoning Zeng, "Research and design of webtop based on could computing," Modern Computer, no. 3, pp. 168-170, 2010

[3] Haifeng Shen, Zhonghua Yang, Chengzheng Sun, "Collaborative web computing: from desktops to webtops," IEEE Distributed Systems Online, vol. 8, no. 4, pp. 3, April 2007, doi:10.1109/MDSO.2007.19

[4] $\mathrm{Li}$ Yan, "Development and application of desktop virtualization technology," Communication Software and Networks (ICCSN), 2011 IEEE 3rd International Conference on, pp.326,329, May 2011, doi: 10.1109/ICCSN.2011.6013725

[5] Feng Wang, Feng Jiang, Chaoyang Li, "Virtual desktop and key technology analysis," Telecommunications Technology, no. 4, pp.24-26, April 2011 